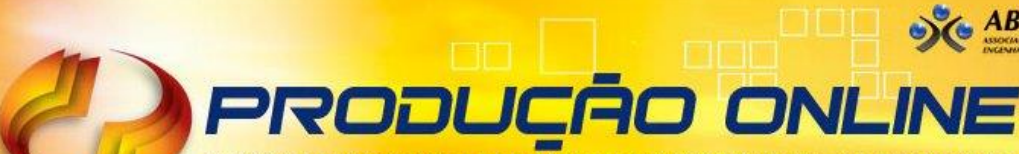 \\ REVISTA CIENTIFICA ELETRÓNICA DE ENGENHARIA DE PRODUÇÃO \\ ISSN 1676-1901
}

\section{A UTILIZAÇ̃̃O DE SISTEMAS DE ERP COMO ANTECEDENTE DA EFICIÊNCIA E EFICÁCIA (INTER)ORGANIZACIONAL: UM ESTUDO EM DIMENSÕES ESTRATÉGICAS EM PEQUENAS E MÉDIAS EMPRESAS ${ }^{1}$}

\section{USE OF ERP SYSTEMS AS ANTECEDENT OF (INTER)ORGANIZATIONAL EFFICIENCY AND EFFECTIVENESS: A STUDY IN STRATEGIC DIMENSIONS IN SMALL AND MEDIUM SIZED ENTERPRISES}

\author{
Renato Borges Fernandes* E-mail: renato@unipam.edu.br \\ Luiz Marcelo Antonialli** E-mail: Imantonialli@dae.ufla.br \\ Custódio Genésio da Costa Filho*** E-mail: custodio@ufv.br \\ Ronaldo Pereira Caixeta* E-mail: ronaldo@unipam.edu.br \\ *Centro Universitário de Patos de Minas (UNIPAM), Patos de Minas, MG \\ ** Universidade Federal de Lavras (UFLA), Lavras, MG \\ *** Universidade Federal de Viçosa (UFV), Viçosa, MG
}

Resumo: Este estudo teve como objetivo analisar o impacto da utilização de sistemas de ERP na eficiência e eficácia organizacional e eficiência interorganizacional de empresas de pequeno e médio portes do município de Patos de Minas. Utilizando-se de um survey adaptado de Saccol et al. (2004), o estudo avaliou as dimensões estratégicas de clientes e consumidores, de fornecedores, de mercado e de rivalidade competitiva e sua relação com eficiência e eficácia organizacionais e com eficiência interorganizacional, quando todas são auxiliadas por sistemas de ERP. Os resultados demonstraram leve relação positiva na explicação da eficiência e eficácia organizacional e um pouco maior na eficiência interorganizacional. Conclui-se que os sistemas de ERP possuem baixa relevância para os aspectos estratégicos em pequenas e médias empresas, confirmando-se a tese da necessidade de maturidade na utilização de sistemas de informação para extrair benefícios estratégicos.

Palavras-chave: Sistemas de informação. Sistemas integrados de gestão. Estratégia empresarial. Pequenas e médias empresas. Mínimos quadrados parciais (PLS).

Abstract: This study aimed to analyze the impact of the use of ERP systems on organizational efficiency and effectiveness and inter-organizational efficiency of small and medium enterprises in Patos de Minas County. Using a survey adapted from Saccol et al. (2004), the study evaluated the strategic dimensions of customers and consumers, suppliers, market and competitive rivalry and their relationship to organizational efficiency and effectiveness and inter-organizational efficiency, all of which aided by ERP systems. The results showed slight positive relationship in explaining the organizational efficiency and effectiveness and a little higher in inter-organizational efficiency. It is concluded that ERP systems have low relevance to the strategic aspects on small and medium enterprises, confirming the thesis of the need to maturity in the use of information systems to extract strategic benefits.

1 Conforme exigências editoriais, os autores confirmam que o estudo é inédito, não foi financiado por qualquer órgão de fomento, não é resultado de dissertação ou tese, não há qualquer conflito de interesses e não foi aprovado por quaisquer comitês de ética. É importante ressaltar que à época da aplicação dos surveys, em 2012, não havia a exigência de tal aprovação. 
Keywords: Information system. Enterprise resource planning. Corporate strategy. Small and medium sized enterprises. Partial least squares (PLS).

\section{INTRODUÇÃO}

As organizações contemporâneas, sejam pequenas, médias ou grandes, estão utilizando, cada vez mais, soluções integradas de tecnologia da informação, seja por enxergarem-nas como ferramentas para eficiência e processos internos (WEITZEN, 1991; BERNROIDER; KOCH, 1999; BECKER et al., 2002), como recurso estratégico ou de sobrevivência (FERREIRA; RAMOS, 2005; SOUZA et al., 2013) ou por modismo tecnológico (WANG, 2001; LEE; COLLAR, 2003; FICHMAN, 2004; SANTOS; MAÇADA, 2010; WANG, 2010; BORT; KIESER, 2011; FELICIANO; MAÇADA, 2013), apesar de não existirem comprovações destas relações de forma geral e sistematizada (BRYNJOLFSSON, 1993; MAHMOOD, 1997; GRAEML, 1998; CAZARINI, 2002; LAURINDO, 2002) ou mesmo com poucas evidências da relação com produtividade e desempenho organizacional (MAHMOOD; SOON, 1991; MAHMOOD, 1997).

Neste sentido e frente às exigências de agilidade relativas ao processo de decisão, sistemas integrados de gestão, ou ERP's (Enterprise Resource Planning), são um recurso cada vez mais utilizado pelas organizações, principalmente, quando os argumentos dos fornecedores são sobrevivência e benefícios tangíveis e estratégicos alcançados pela sua utilização, calcados na eficiência e eficácia organizacionais e interorganizacionais. Souza et al. (2013) observam que quando esses argumentos se aliam à visão dos empresários sobre potenciais benefícios de eficiência em processos, análise de benchmarking, passagem de visão departamental para processos e maior controle e inovação, fazem com este mercado seja crescente.

Apesar do desaquecimento no mercado de ERP's entre 2001 e 2003 (SACCOL et al., 2004), Macêdo, Gaete e Joia (2014) afirmam que este é o mercado que mais cresceu em 2011 na indústria de tecnologia da informação, principalmente no segmento de pequenas e médias empresas. A tendência de que as desenvolvedoras desse tipo de solução apontassem seu foco para o mercado de pequenas e médias empresas (PME) já havia sido observada (BARROS, 2003; 
MESQUITA, 2003) e demonstra saturação, em termos de vendas, para o mercado de grandes empresas.

Apesar da tendência para PME's e do crescimento apontado, Sherman (2000, p. 52) argumenta que "muitas são as empresas desencantadas com o ERP", por diversos motivos: times de suporte inexperientes, acarretado por alta demanda e, consequentemente, falta de tempo hábil para treinamento; processo de implementação longo, podendo chegar a três anos; nova estrutura de negócios, com possibilidades de perda de posições para funcionários; custos elevados com consultorias e treinamento de usuários e, muitas vezes, indo além do previsto; alta complexidade de customização de alguns módulos; expectativas de benefícios que nem sempre se concretizam. Para Scheer e Habermann (2000), a maior causa de insatisfação das compradoras é a escala de reengenharia dos processos envolvidos e tarefas de customização durante o processo de implementação. O processo de implementação e serviços associados custam às empresas três a sete vezes mais do que a compra da licença do software.

Com todos esses desafios, a implantação e utilização de sistemas ERP exigem alto grau de maturidade da organização em termos de organização e processos, o que poderia ser um dificultador para médias e, principalmente, pequenas empresas, especialmente quando utilização e benefícios do sistema transpassam de atividades operacionais para estratégicas.

Assim, verifica-se que, cada vez mais, são necessárias avaliações dos benefícios que este tipo de solução pode trazer para as empresas em todas as suas áreas, seja operacional, gerencial ou estratégica. Todos estes aspectos remetem a uma oportunidade de estudo: quais os impactos na eficiência e eficácia de organizações de pequeno e médio portes adotantes deste tipo de solução, tendo como base a dimensão estratégica? Como são poucos os estudos sobre seus impactos na dimensão estratégica (MENDES; ESCRIVÃO FILHO, 2000; 2002; SACCOL et al., 2004), optou-se por pesquisar sua relação com algumas variáveis estratégicas.

Assim, o presente estudo tem por objetivo verificar a relação entre utilização de sistemas de ERP e sua influência nas variáveis estratégicas clientes e consumidores, fornecedores, mercado e rivalidade competitiva e como estas variáveis impactam na eficiência e eficácia organizacional e eficiência 
interorganizacional em pequenas e médias empresas de Patos de Minas e região que implantaram Sistemas ERP há mais de um ano, com base no modelo de avaliação estratégica de tecnologias proposto, inicialmente, por Mahmood e Soon (1991) e alterado para avaliação de sistemas de ERP por Saccol et al. (2004).

\section{REFERENCIAL TEÓRICO}

\subsection{Sistemas Integrados de Gestão - ERP}

ERPs possuem ampla literatura que demonstram suas origens e evoluções, os conceitua e demonstra seus diversos aspectos nas organizações (DAVENPORT, 1998; WOOD JR., 1999; KUMAR; HILLEGERSBERG, 2000; SOUZA; ZWICKER, 2000; MENDES; ESCRIVÃO FILHO, 2000; 2002; SACCOL et al., 2004).

Para O'Brien (2002, p. 174), ERP é um conceito amplo, sendo um processo de planejamento de recursos empresariais, auxiliado por sistema de ERP, no qual, "em lugar de se concentrar em requisitos de processamento de informações de funções organizacionais, [...] se concentra no apoio a processos empresariais envolvidos nas operações de uma empresa". A utilização de um sistema que possui conceito de integração, então, "pode produzir benefícios quantitativos e qualitativos, tais como maior produtividade, eficiência, satisfação do usuário/cliente, rapidez de tomada de decisão, redução de custos, controle gerencial, inovação tecnológica" (SANTOS, 2010, p. 169).

Scheer e Habermann (2000) argumentam, todavia, que a implantação de sistemas para o usuário é geralmente cara e rodeada de incertezas, tais como seleção de ferramentas apropriadas, duração do ciclo de implantação ou própria dificuldade envolvida na avaliação dos custos. Stamford (2003) reuniu, em seu estudo, pesquisas empíricas que demonstram que entre 1/2 e 2/3 dos sistemas de informações projetados falham e que $31 \%$ destes projetos são cancelados antes de estarem finalizados.

Verifica-se, então, um paradoxo: enquanto diversos autores propõem benefícios potenciais, pesquisas demonstram gastos excessivos com projetos, muitas vezes inadequados. Logicamente, faz-se necessária, então, uma avaliação dos benefícios alcançados com projetos de implantação concluídos. Hedman e 
Borell (2002) e Sedera, Gable e Chan (2003) até demonstraram importância vital da avaliação do impacto dos sistemas ERP sobre a efetividade organizacional, apesar da associação complicada, devido à própria amplitude dos sistemas integrados de gestão. Nas pequenas e médias empresas, esta relação é ainda mais criticada, com argumentação de que a utilização de sistemas integrados de gestão só será bem aproveitada em organizações com divisões e processos bem estabelecidos e com maturidade de gestão para entender e buscar seus benefícios (CHAN, SABHERWAL; TATCHER, 2006; LÖBLER; BOBSIN; VISENTINI, 2008).

Da mesma forma, Davenport (1998) argumenta que um dos temas menos abordados pelos sistemas de ERP é a verificação de seus resultados na estratégia organizacional. Neste sentido, Saccol et al. (2004) procuraram entender esta relação nas maiores empresas do Brasil. Entretanto, em pequenas e médias empresas (PME's), a lacuna apontada por Davenport (1998) é ainda mais reforçada, existindo poucos trabalhos que abordam o tema (MENDES; ESCRIVÃO FILHO, 2000; 2002). Tal fato aponta para a necessidade de estudos que verifiquem sua eficácia para a estratégia de organizações de pequeno e médio porte.

\subsection{Caráter estratégico de sistemas de ERP em pequenas e médias empresas}

A literatura com visão positiva das tecnologias da informação e que demonstra potencial de ganhos para empresas é ampla (WEITZEN, 1991; DAVENPORT, 1998; O'BRIEN, 2001; LAURINDO, 2002; LAUDON; LAUDON, 2004). Contudo, são diversos os autores críticos desta visão, que perpassam pelo modismo e consumerização (WOOD JR., 1999; LEE; COLLAR, 2003; WANG, 2001; 2010; BORT; KIESER, 2011; FELICIANO; MAÇADA, 2013), pelo mimetismo no abandono de tecnologias (SANTOS; MAÇADA, 2010) e, em artigo polêmico, pela transformação das tecnologias da informação em commodities (CARR, 2003). Neste sentido, Luftman, Lewis e Oldach (1993) já entendiam que TI, unicamente, não fornece vantagem competitiva duradoura.

Diferentemente, para Kumar e Hillegersberg (2000) a utilização de sistemas de ERP é, atualmente, uma questão de necessidade para entrada e continuidade em determinado mercado e, como visto na seção 2.1 , a relação entre sistemas de ERP e estratégia ainda é incipiente, justificando sua investigação. 
Sobre esta relação, Parsons (1983) desenvolveu um modelo de avaliação da TI na competitividade, encontrando três níveis de impacto: na indústria como um todo, alterando produtos e serviços, mercados e economia de produção; na firma, com modificações nas forças competitivas e; na estratégia, afetando estratégias de baixo custo, diferenciação de produtos e nichos. Outros autores, como Bakos e Treacy (1986) e Clemons (1987) também já haviam desenvolvido modelos de avaliação que classificavam o impacto da tecnologia da informação na estratégia corporativa nos níveis interno (eficiência e eficácia de estruturas e processos organizacionais para atingir objetivos e metas), competitivo (manobras competitivas na indústria em que atua), de portfólio de negócio (escolhas de posicionamento na indústria) e em enfoque interno (eficiência) e externo (valor para o cliente), respectivamente.

Mahmood e Soon (1991), entretanto, criticam estes modelos, argumentando que faltam testes empíricos que os comprovem, além de não haver modelos explanatórios decorrentes, o que afetaria, significativamente, a compreensão do impacto da TI na estratégia ou competitividade. Desta forma, os autores conduziram pesquisa empírica com as 500 maiores empresas dos EUA, com rigorosos testes e validação, para desenvolvimento de um modelo de avaliação da relação $\mathrm{TI} x$ variáveis estratégicas, que é composto das seguintes dimensões: compradores e consumidores, produtos e serviços, rivalidade competitiva, economia de produção, estrutura de custo e capacidade, fornecedores, eficiência interna da organização, custo de busca e de mudança, eficiência interorganizacional e mercado e formação de preço. O modelo de Mahmood e Soon (1991) possui fortes bases nas cinco forças competitivas propostas por Porter (1979), apesar de haver crítica à não comprovação empírica sobre a relação direta entre estratégia/competitividade e tecnologia da informação.

Por sua vez, Saccol et al. (2004) adaptaram, de forma sistemática e rigorosa, o instrumento criado por Mahmood e Soon (1991), especificamente para avaliação do impacto de ERPs em variáveis estratégicas, o que resultou na redução de 50 para 34 questões e de 10 para 7 dimensões.

A dimensão clientes e consumidores trata de como os sistemas de ERP devem auxiliar no relacionamento entre clientes/consumidores e empresa, devendo estes suportar, com informações, negócios eletrônicos (e-business). Trata-se aqui, 
tanto de informações sobre empresa e produtos, como de suporte administrativo. Segundo Thompson e Strickland (2002), esta pode ser uma forma de entregar um valor superior aos consumidores e clientes e, supostamente, gerando vantagem competitiva para a empresa. Além disso, conforme Swift (2001), sistemas de ERP auxiliam na implantação de estratégias e sistemas de relacionamento com clientes (CRM), que, por sua vez, auxiliam na estratégia organizacional.

$\mathrm{Na}$ dimensão "fornecedores", sistemas de ERP devem desempenhar triplo papel: auxiliar na gestão de compras, aproximar fornecedores e empresa e colaborar na relação do poder de barganha da empresa. Em relação à gestão de compras, Mahmood e Soon (1991) destacam o auxílio nas decisões de produção e compra. A aproximação entre empresa e fornecedores acontece na agilização de acesso aos pedidos, redução do tempo entre o pedido e a disponibilização para uso de um produto (lead time) e monitoramento da qualidade de produtos/serviços recebidos pelos fornecedores. No que diz respeito ao poder de barganha, sistemas de ERP devem contribuir para o seu aumento junto aos fornecedores, o que acontece pela gestão de informações referente aos mesmos, bem como auxiliar na busca de novos fornecedores.

Quanto à dimensão "mercado", sistemas de ERP devem contribuir para a relação entre empresa e mercado, auxiliando em previsões de tendências, vendas e necessidades dos clientes, de forma geral; identificação de ações visando à lealdade dos clientes e contribuindo para redução de custos de marketing, ao mesmo tempo em que o torna mais eficaz.

No que tange à dimensão "rivalidade competitiva", Mahmood e Soon (1991) propõem que as tecnologias da informação auxiliem nos processos de concorrência e obtenção de vantagem competitiva. Desta forma, sistemas de ERP deverão contribuir para a obtenção de informações relevantes sobre concorrentes e processos de concorrência, além de auxiliar na criação de produtos/serviços não imitáveis ou substitutos mais rapidamente.

A dimensão de eficiência e eficácia organizacional está relacionada à maneira como sistemas de ERP contribuirão, em especial, para a gestão organizacional, agregando valor aos seus diversos aspectos, principalmente nos processos de tomada de decisão e seus meios, como reuniões e discussões e seus conteúdos. Além disso, o sistema deverá melhorar, de forma geral, a coordenação entre as 
áreas interfuncionais da empresa, avaliação orçamentária, elaboração do planejamento estratégico e aumentar a participação de mercado e margem de lucro da empresa (MAHMOOD; SOON, 1991).

Por fim, a dimensão "eficiência interorganizacional" trata do relacionamento estratégico com outras organizações, sejam elas da própria empresa (filiais, subsidiárias etc.), sejam externas. Conforme Mahmood e Soon (1991), a TI pode auxiliar na padronização de comunicação e coordenação de atividades de escopo regional ao global em unidades próprias da empresa, coordenação de atividades com clientes e fornecedores e agregar mais informações aos produtos e serviços da empresa.

Observa-se que as duas últimas dimensões (eficiência e eficácia organizacional e eficiência interorganizacional) podem ser consideradas dimensões de resultado (HEDMAN; BORELL, 2002; SEDERA; GABLE; CHAN, 2003), podendo ser explicadas pelas outras dimensões. Este é o tema deste estudo.

\section{METODOLOGIA}

Visando à consecução dos objetivos foi conduzida pesquisa de abordagem quantitativa junto a empresas que possuíam sistemas de ERP implantados no município de Patos de Minas, respeitando-se os seguintes critérios: i) possuíssem mais de um ano de implantação do sistema; e ii) tivessem implantados módulos de compra, vendas e gerencial, pois estão relacionados às atividades meio, conforme Santos (2010), considerando que as dimensões analisadas estão intimamente ligadas a estes módulos.

De acordo com os constructos levantados nas referências teóricas, propõemse oito hipóteses em relação à Eficiência e Eficácia Organizacional e Eficiência Interorganizacional:

H1: há relação positiva entre os constructos "clientes e consumidores" e "eficiência e eficácia organizacional", quando auxiliados por sistemas ERP.

H2: há relação positiva entre os constructos "fornecedores" e "eficiência e eficácia organizacional", quando auxiliados por sistemas ERP.

H3: há relação positiva entre os constructos "mercado" e "eficiência e eficácia organizacional”, quando auxiliados por sistemas ERP. 
H4: há relação positiva entre os constructos "rivalidade competitiva" e "eficiência e eficácia organizacional", quando auxiliados por sistemas ERP.

H5: há relação positiva entre os constructos "clientes e consumidores" e "eficiência interorganizacional", quando auxiliados por sistemas ERP.

H6: há relação positiva entre os constructos "fornecedores" e "eficiência interorganizacional", quando auxiliados por sistemas ERP.

H7: há relação positiva entre os constructos "mercado" e "eficiência interorganizacional”, quando auxiliados por sistemas ERP.

H8: há relação positiva entre os constructos "rivalidade competitiva" e "eficiência interorganizacional", quando auxiliados por sistemas ERP.

Os constructos e hipóteses estão alicerçados teoricamente nas dimensões propostas por Mahmood e Soon (1991) e Saccol et al. (2004). Suas relações foram dispostas para explicar eficiência e eficácia organizacional e interorganizacional com base em Hedman e Borell (2002) e Sedera, Gable e Chan (2003), as quais podem ser visualizadas na Figura 1.

Figura 1 - Modelos propostos para o teste das hipóteses

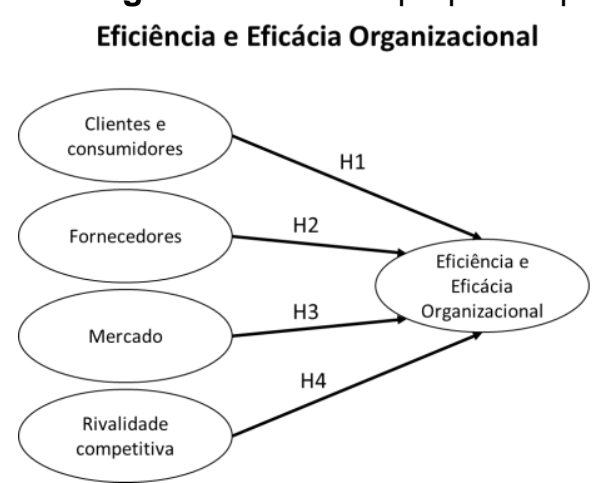

Eficiência Interorganizacional

Fonte: Elaborado pelos autores

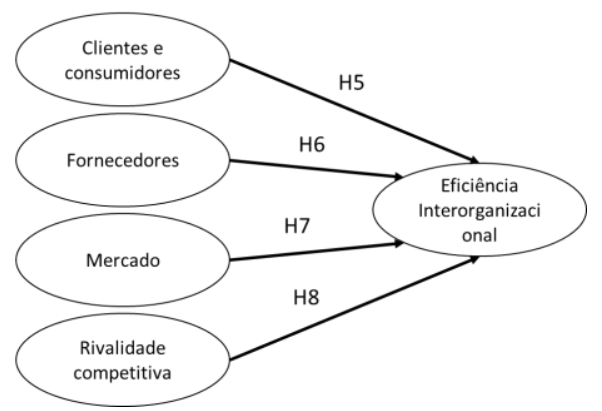

Para verificação das hipóteses, foi conduzida pesquisa de campo com aplicação de versão adaptada do survey proposto por Saccol et al. (2004). Este instrumento foi traduzido e adaptado a partir do modelo proposto por Mahmood e Soon (1991) e foi escolhido devido ao rigoroso processo de validação deste autor e de adaptação por Saccol et al. (2004). Do conjunto de 34 questões em 7 dimensões, foi retirada a dimensão "Produção", por haver vieses relacionados às organizações estudadas. Desta forma, restaram 6 dimensões com 28 questões parametrizadas por escala tipo Likert de 7 pontos, que derivou de 1, discorda totalmente, a 7 , concorda totalmente (Quadro 1). Também, foram acrescidas quatro questões de 
controle, quais sejam: porte da empresa, ramo de negócios, módulos implantados e tempo de implantação.

A população foi pesquisada e definida junto às softhouses que atuam no mercado do município e consistiu de um total de 37 empresas, já considerados os critérios preestabelecidos. Nas empresas, foram identificados 153 gerentes e diretores que estavam relacionados às dimensões estratégicas propostas. Foi utilizada uma amostra probabilística simples, acarretando na seleção de 109 gerentes e diretores em 30 empresas. A margem de erro foi estimada em $5 \%$, com confiabilidade em $95 \%$.

Em seguida, foi realizado estudo preliminar para averiguar adequabilidade das perguntas e escala do questionário. Este estudo contou com especialistas no assunto que avaliaram, criticaram e sugeriram alterações. Após esta fase, o questionário foi testado com 10 sujeitos definidos na amostra, com o objetivo de avaliarem relevância, clareza e compreensão das questões. Assim, conseguiu-se um questionário mais confiável, resultando em respostas com maior credibilidade.

Os questionários foram enviados por e-mail aos sujeitos selecionados na amostra junto às softhouses, com suas respostas já sendo armazenadas em banco de dados online. Eles continham objetivos e instruções de preenchimento, permitindo autocondução pelos sujeitos pesquisados. Esta fase ocorreu entre 13/11 e 25/12/2012.

Para a análise foi utilizada a técnica de modelagem de equações estruturais que, conforme Hair Jr. et al. (2014), permite utilizar múltiplas técnicas de regressão e análise fatorial simultaneamente. Foi utilizada a técnica de PLS-PM, pelo software SmartPLS, que, conforme Hair Jr. et al. (2014), emprega método de regressão que maximiza a variância explicada. Esta técnica foi escolhida por alguns fatores: i) conforme Henseler, Ringleand e Sinkovics (2009), por utilizar o método de reamostragem, permite aplicação em amostras menores do que outras técnicas, que é o caso deste estudo e; ii) não exige normalidade nas distribuições dos dados. 
Quadro 1 - Variáveis agrupadas por suas dimensões

\section{CLIENTES E CONSUMIDORES}

C1. O sistema contribui para a disponibilização do banco de dados e informações da empresa para os clientes.

C2. O sistema auxilia a empresa a prover suporte administrativo aos clientes.

\section{RIVALIDADE COMPETITIVA}

RC1. O sistema contribui diretamente na investida contra competidores através de informações relevantes.

RC2. O sistema contribui para a criação de produtos/serviços não imitáveis. RC3. O sistema facilita os processos de concorrência.

\section{FORNECEDORES}

\section{MERCADO}

F1. O sistema colabora para o aumento do poder de barganha da empresa junto aos seus fornecedores.

F2. O sistema facilita aos fornecedores o acesso aos pedidos da empresa.

F3. O sistema auxilia em reduzir a incerteza do lead time.

F4. O sistema contribui para se encontrar novas alternativas de fornecedores.

F5. O sistema auxilia nas decisões relativas à produção x compra de insumos.

F6. O sistema auxilia o monitoramento da qualidade de produtos e serviços recebidos dos fornecedores

\section{EFICIÊNCIA E EFICÁCIA ORGANIZACIONAL}

EEO1. O sistema auxilia a melhorar o processo

e o conteúdo das decisões da organização.

EEO2. O sistema melhora as reuniões e

discussões internas provendo-as de

informações adequadas.

EEO3. O sistema possibilita melhor coordenação entre as áreas funcionais da empresa.

EEO4. O sistema contribui para melhorar avaliações nos relatórios anuais do orçamento.

EEO5. O sistema melhora a elaboração do planejamento estratégico da empresa.

EEO6. O sistema auxilia a aumentar a margem de lucro da empresa.

EEO7. O sistema apresenta contribuições para o aumento da participação de mercado da empresa.

Fonte: Adaptado de Saccol et al. (2004, p. 29)

Para análise dos modelos estruturais, foram seguidos os sequenciais procedimentais propostos por Bido, Silva e Ringle (2014): 1) avaliação dos modelos de mensuração, utilizando-se de 1.1) análise das variâncias médias extraídas (AVE's) de cada variável latente (VL) (dimensão estratégica), 1.2) validação

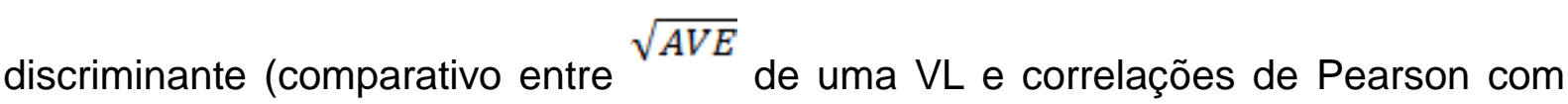


outras VLs), 1.3) indicadores de confiabilidade (alfa de Cronbach e confiabilidade composta), 1.4) comparativo de média com teste t Student entre valores originais e valores gerados das variáveis observadas (VOs) pela técnica de reamostragem (bootstrapping); 2) avaliação do modelo estrutural utilizando-se de 2.1) comparativo de média com teste t Student entre valores originais e valores gerados das variáveis latentes (VLs) pela técnica de reamostragem, 2.2) avaliação da percentagem de variância explicada pelo coeficiente de determinação de Pearson $\left.\left(R^{2}\right), 2.3\right)$ indicador de relevância preditiva do modelo estrutural de Stone-Geisser $\left(Q^{2}\right)$ e 2.4) indicador do tamanho do efeito do modelo estrutural pelo indicador de Cohen $\left(\mathrm{f}^{2}\right)$.

Para análise das hipóteses foram avaliados os coeficientes de caminho ( $\ulcorner$ ) de cada relação proposta para sua confirmação ou rejeição.

\section{RESULTADOS}

Em relação ao perfil, das 30 empresas que participaram do estudo, a maioria $(82,6 \%)$ são de comércio, seguidas de serviço (11\%) e indústria $(6,4 \%)$. A maior parte delas é de médio porte $(70,9 \%)$, com restante de pequeno porte $(29,1 \%)$. Os ramos de negócio foram os mais variados: automobilístico, comércio de calçados, clube, comércio de eletrodomésticos, farmácias, fotografia, genética suína, informática, instituição de ensino superior, insumos agrícolas, indústria de laticínios, materiais para construção, indústria de armações metálicas, panificadoras, papelarias, restaurante, supermercado e transporte público.

Os resultados foram agrupados em cada dimensão estratégica, conforme seções a seguir.

\subsection{Eficiência e Eficácia Organizacional}

Para verificação das relações em cada hipótese proposta foi realizada análise de caminhos pois, conforme Hair Jr. et al. (2014), é por meio desta análise que se demonstram relações de dependência e causa e efeito entre constructos, além da força entre estas relações. Para melhorar esta relação, foram retiradas as variáveis observadas (VOs) com cargas fatoriais menores que 0,6. Assim, após primeiro teste, 
foram eliminadas F1 e F2 do constructo independente "fornecedores" e EEO7 do constructo dependente Eficiência e Eficácia Organizacional.

Para validação do modelo, foi seguido o fluxo de análises proposto por Bido, Silva e Ringle (2014). Desta forma, inicialmente, procedeu-se a avaliação dos modelos de mensuração, pela análise das variâncias médias extraídas (AVEs), alfa de Cronbach (AC) e confiabilidade composta (CC). Os dados referentes a esta análise podem ser visualizados na Tabela 1.

Tabela 1 - Valores iniciais de qualificação do modelo de equação estrutural

\begin{tabular}{lllll}
\hline Dimensões & AVE & $\begin{array}{l}\text { Alfa de } \\
\text { Cronbach }\end{array}$ & $\begin{array}{l}\text { Confiabilidade } \\
\text { composta }\end{array}$ & $\mathbf{R}^{\mathbf{2}}$ \\
\hline Clientes e Consumidores & 0,917 & 0,910 & 0,957 & - \\
Eficiência e Eficácia & & & & 0,834 \\
Organizacional & 0,711 & 0,917 & 0,936 & - \\
Fornecedores & 0,659 & 0,831 & 0,885 & - \\
Mercado & 0,676 & 0,901 & 0,925 & - \\
Rivalidade Competitiva & 0,780 & 0,867 & 0,914 &
\end{tabular}

Fonte: Dados da pesquisa (2012)

Obs.: Variáveis independentes não possuem $\mathrm{R}^{2}$.

Hair et al. (2014) argumentam que a AVE demonstra a porção média compartilhada de variância entre VL e suas VOs. Para Henseler, Ringleand e Sinkovics (2009), a análise das AVEs se dá por meio do critério de Fornell e Larcker (1981), que sugere valores maiores que 0,5. No caso deste modelo estrutural, todas as AVEs estão acima de 0,5, demonstrando que as VOs estão explicando, adequadamente, cada constructo.

Em relação às medidas de confiabilidade, para Bido, Silva e Ringle (2014, p. 65), deve-se priorizar a CC na técnica de PLS-PM, ao invés do AC, pois, aquela "prioriza as variáveis de acordo com as suas confiabilidades, enquanto o AC é muito sensível ao número de variáveis em cada constructo". Neste estudo, optou-se pela análise de ambos os indicadores. Conforme Hair Jr. et al. (2014), valores acima de 0,6 são satisfatórios para $A C$ e acima de 0,7 para CC, considerando pesquisas exploratórias. Conforme Tabela 1, todos os constructos possuem consistência interna em ambos os indicadores de confiabilidade, segundo os parâmetros propostos por Hair Jr. et al. (2014).

O próximo passo é a validação discriminante do modelo que, conforme Hair Jr. et al. (2014), é o que demonstra que cada VL é distinta das outras. Para sua verificação, Fornell e Lacker (1981) apresentam um método de comparação da raiz 
quadrada da AVE $\left({ }^{\sqrt{A V E}}\right.$ ) de cada VL com suas respectivas correlações de Pearson com as outras VLs. Para os autores, há validade discriminante quando a ${ }^{\sqrt{A V E}}$ for maior do que quaisquer correlações com outras VLs, independentemente, da diferença. No modelo em questão, em primeira análise, a VL Eficiência e Eficácia Organizacional teve sua ${ }^{\sqrt{A V E}}(0,843)$ menor do que sua correlação com a VL Mercado (0,864). Bido, Silva e Ringle (2014) demonstram que sendo uma diferença baixa $(\sim 2,5 \%)$, poder-se-ia optar por considerar o modelo tal qual está, mas, se se quer rigor, as VOs com as menores cargas fatorais devem ser retiradas do modelo, visando a elevar a AVE da respectiva VL. Neste caso, optou-se pela retirada da VO M6, com menor carga fatorial dentre as demais $(0,680)$, conferindo mais ajuste e rigor ao modelo. Os valores ajustados podem ser visualizados na Tabela 2.

Tabela 2 - Valores ajustados de qualificação discriminante do modelo estrutural

\begin{tabular}{llllll}
\hline Dimensões & 1 & 2 & 3 & 4 & 5 \\
\hline 1. Clientes e Consumidores & 0,958 & & & & \\
2. Eficiência e Eficácia & & & & & \\
Organizacional & 0,789 & 0,843 & & & \\
3. Fornecedores & 0,514 & 0,694 & 0,812 & & \\
4. Mercado & 0,639 & 0,805 & 0,743 & 0,866 & \\
5. Rivalidade Competitiva & 0,827 & 0,707 & 0,619 & 0,655 & 0,883 \\
\hline
\end{tabular}

Fonte: Dados da pesquisa (2012)

Obs.: valores em negrito representam a raiz quadrada da AVE da VL. Os outros valores são correlações de Pearson entre cada variável.

Em seguida, conforme proposto por Bido, Silva e Ringle (2014), empreendeuse a avaliação do modelo estrutural, iniciando-se pela avaliação dos coeficientes de determinação de Pearson ( $R^{2}$ ). Segundo Cohen (1988 apud BIDO; SILVA; RINGLE, 2014, p. 67), este coeficiente demonstra "a porção de variância das variáveis endógenas que é explicada pelo modelo estrutural" e deve possuir, em ciências sociais e comportamentais, $26 \%$ ou mais para ser considerada como porção de efeito grande. Verificou-se, após ajustes anteriores, que as VLs independentes estão explicando 79,2\% da VL Eficiência e Eficácia Organizacional, um valor considerado significativo.

Deve-se verificar, então, se as correlações entre VLs e suas VOs e regressões das VLs independentes com a VL dependente são significantes $\left({ }^{p \leq 0,05}\right)$. Conforme Bida, Silva e Ringle (2014), esta verificação é realizada pela Revista Produção Online, Florianópolis, SC, v.15, n. 4, p. 1351-1376, out./dez. 2015. 
comparação das médias, por meio de testes t de Student, entre os valores originais das observações e aqueles originados pela técnica de reamostragem. Devem ser considerados valores acima de 1,96 , que resultam em ${ }^{p \text { valores }} \leq 0,05$. No modelo estrutural sobre Eficiência e Eficácia Organizacional, todos os ${ }^{p}$ valores foram maiores que 1,96, demonstrando, conforme Tabela 3, que todas as correlações e regressões do modelo são significativas $\left({ }^{p \leq 0,05}\right)$.

Seguindo a análise do modelo, conforme Bido, Silva e Ringle (2014, p. 69), devem ser avaliados mais dois indicadores de qualidade de ajuste. São eles o indicador de "relevância preditiva $\left(\mathrm{Q}^{2}\right)$, ou indicador de Stone-Geisser e o indicador de tamanho do efeito $\left(\mathrm{f}^{2}\right)$ ou indicador de Cohen. $O \mathrm{Q}^{2}$ avalia a capacidade de predição (acurácia) do modelo ajustado. Conforme Hair Jr. et al. (2014), um valor igual a um demonstra que o modelo prevê, sem erros, a realidade. Já o f² é a razão entre a parte explicada pelo modelo $\left(R^{2}\right)$ e a não explicada $\left(1-R^{2}\right)$ e avalia o "quanto cada constructo é útil para o ajuste do modelo" (BIDO, SILVA; RINGLE, 2014, p. 70). Conforme Hair Jr. et al. (2014), valores de $f^{2}$ são considerados grandes a partir de 0,35 .

Tabela 3 - Valores t de Student e p-valores

\begin{tabular}{|c|c|c|c|c|c|}
\hline Variáveis latentes & Teste $t$ & p-valor & $\begin{array}{l}\text { Variáveis } \\
\text { observadas }\end{array}$ & Teste $t$ & $\mathrm{p}$-valor \\
\hline Clientes e & \multirow{4}{*}{3,879} & \multirow{4}{*}{0,000} & C1 & 112,434 & 0,000 \\
\hline \multirow{3}{*}{ Consumidores } & & & $\mathrm{C} 2$ & 126,090 & 0,000 \\
\hline & & & EEO1 & 26,167 & 0,000 \\
\hline & & & EEO2 & 17,499 & 0,000 \\
\hline \multirow{5}{*}{$\begin{array}{l}\text { Eficiência e Eficácia } \\
\text { Organizacional }\end{array}$} & \multirow{5}{*}{ - } & \multirow{5}{*}{-} & EEO3 & 35,560 & 0,000 \\
\hline & & & EEO4 & 13,023 & 0,000 \\
\hline & & & EEO5 & 47,244 & 0,000 \\
\hline & & & EEO6 & 32,223 & 0,000 \\
\hline & & & F3 & 8,342 & 0,000 \\
\hline \multirow{5}{*}{ Fornecedores } & \multirow{5}{*}{2,222} & \multirow{5}{*}{0,027} & F4 & 22,242 & 0.000 \\
\hline & & & F5 & 33,805 & 0,000 \\
\hline & & & F6 & 9,772 & 0.000 \\
\hline & & & M1 & 50,537 & 0,000 \\
\hline & & & $\mathrm{M} 2$ & 40,881 & 0,000 \\
\hline \multirow[t]{4}{*}{ Mercado } & \multirow[t]{4}{*}{4,404} & \multirow[t]{4}{*}{0,000} & M3 & 10,712 & 0,000 \\
\hline & & & M4 & 55,789 & 0,000 \\
\hline & & & M5 & 11,173 & 0,000 \\
\hline & & & RC1 & 69,712 & 0,000 \\
\hline \multirow[t]{2}{*}{ Rivalidade Competitiva } & \multirow[t]{2}{*}{2,001} & \multirow[t]{2}{*}{0,031} & $\mathrm{RC2}$ & 24,919 & 0,000 \\
\hline & & & RC3 & 24,841 & 0,000 \\
\hline
\end{tabular}

Fonte: Dados da pesquisa (2012) 
No caso do modelo de explicação de Eficiência e Eficácia Organizacional, estes valores estão dentro do padrão e demonstram que os constructos possuem acurácia $\left(Q^{2}=0,551\right)$ e são relevantes $\left(f^{2}\right)$ para o ajuste geral do modelo (Tabela 4$)$.

Tabela 4 - Valores de validade preditiva $\left(\mathrm{Q}^{2}\right)$ e de tamanho do efeito $\left(\mathrm{f}^{2}\right)$

\begin{tabular}{lll}
\hline Dimensões & $\mathrm{Q}^{2}$ & $\mathrm{f}^{2}$ \\
\hline Clientes e Consumidores & - & 0,632 \\
Eficiência e Eficácia & & \\
Organizacional & 0,551 & 0,589 \\
Fornecedores & - & 0,426 \\
Mercado & - & 0,622 \\
Rivalidade Competitiva & - & 0,536
\end{tabular}

Fonte: Dados da pesquisa (2012)

Por fim, Bido, Silva e Ringle (2014) apontam a necessidade de avaliar um indicador de ajuste geral do modelo. Para tal, Tenenhuaus et al. (2005) propuseram o índice de adequação do modelo (Goodness of fit - GoF), calculado pela média geométrica entre $\mathrm{R}^{2}$ e média ponderada das $\mathrm{AVEs}$ de cada VL, com valor aceitável, para ciências sociais e do comportamento, acima de 0,36 , conforme indicado por Wetzels et al. (2009 apud BIDO; SILVA; RINGLE, 2014).

Tabela 5 - Índice de adequação do modelo estrutural (GoF)

\begin{tabular}{|c|c|c|c|c|}
\hline \multirow{2}{*}{ Dimensões } & \multirow[b]{2}{*}{ Variáveis } & \multirow[t]{2}{*}{ AVE } & \multirow{2}{*}{$\begin{array}{l}\text { AVE } x \\
\text { Variáveis }\end{array}$} & \multirow[t]{2}{*}{$\mathrm{R}^{2}$} \\
\hline & & & & \\
\hline $\begin{array}{l}\text { Clientes e Consumidores } \\
\text { Eficiência e Eficácia }\end{array}$ & 2 & 0,917 & 1,834 & \\
\hline Organizacional & 6 & 0,711 & 4,266 & 0,792 \\
\hline Fornecedores & 4 & 0,659 & 2,636 & \\
\hline Mercado & 5 & 0,751 & 3,755 & \\
\hline \multirow[t]{4}{*}{ Rivalidade Competitiva } & 3 & 0,780 & 2,340 & \\
\hline & & & AVE Médio & $\mathrm{R}^{2}$ Médio $\left.{ }^{(*}\right)$ \\
\hline & & & 0,74155 & 0,792 \\
\hline & & & $\mathrm{GoF}=0,766$ & \\
\hline
\end{tabular}

Fonte: Dados da pesquisa (2012)

$\mathrm{GoF}=0,76636$

Obs.: $\left(^{*}\right)$ Média ponderada pelo número de variáveis. $\left.{ }^{(*}\right)$ Média aritmética

Para o modelo estrutural de Eficiência e Eficácia Organizacional foi encontrado o valor de 0,76636, o qual demonstra excelente adequação do modelo estrutural. O modelo final para Eficiência e Eficácia Organizacional, com cargas fatoriais $(\lambda)$, betas das regressões $(\beta)$ e coeficientes de determinação $\left(R^{2}\right)$ pode ser visto na Figura 2. 
Figura 2 - Modelo estrutural ajustado sobre Eficiência e Eficácia Organizacional

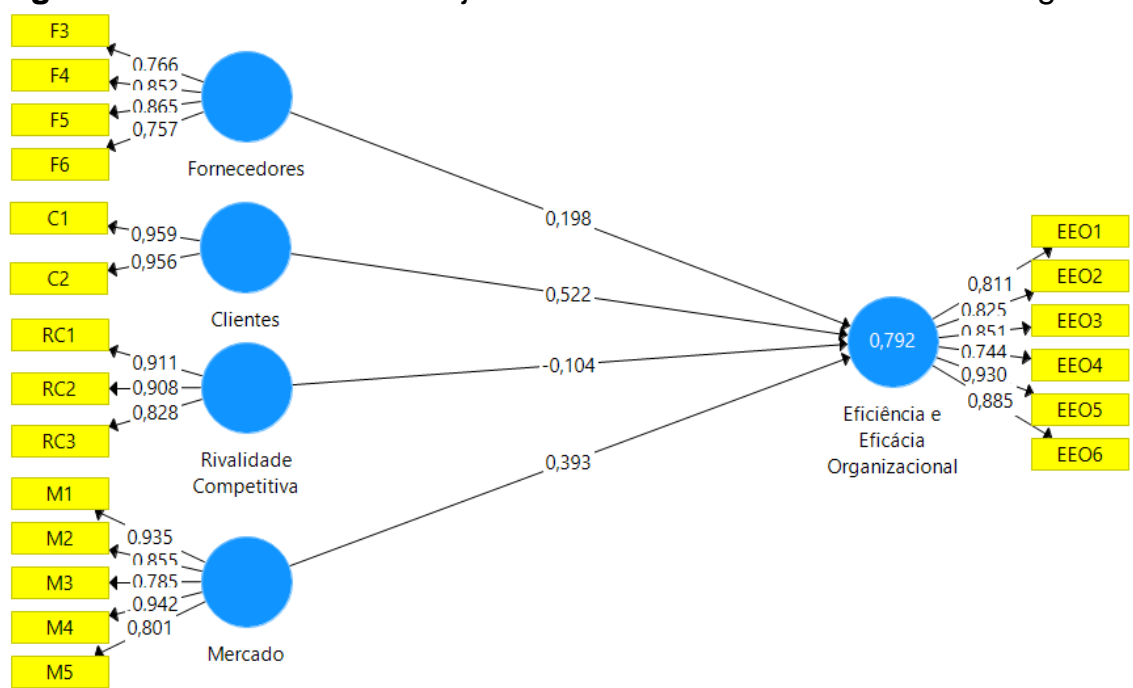

Fonte: Dados da pesquisa (2012)

\subsection{Eficiência Interorganizacional}

Após análise do modelo estrutural para Eficiência e Eficácia Organizacional, empreendeu-se avaliação e ajustes no modelo para Eficiência Interorganizacional. Inicialmente, foram retiradas VOs com cargas fatoriais abaixo de 0,6 (HAIR JR. et al., 2014), sendo elas F1 e F2, relativas ao constructo independente Fornecedores. Em seguida, foram analisadas as AVEs, confiabilidades compostas e alfas de Cronbach em cada constructo. Todos os indicadores são satisfatórios, segundo os padrões propostos (AVE > 0,5, alfas de Cronbach > 0,6 e confiabilidades compostas $>0,7)$, demonstrando que as VOs explicam adequadamente os constructos e que há consistência interna em cada um deles (HAIR JR. et al., 2014).

Tabela 6 - Valores de qualificação do modelo de equação estrutural

\begin{tabular}{lllll}
\hline Dimensões & AVE & $\begin{array}{l}\text { Alfa de } \\
\text { Cronbach }\end{array}$ & $\begin{array}{l}\text { Confiabilidade } \\
\text { composta }\end{array}$ & $\mathrm{R}^{2}$ \\
\hline Clientes e Consumidores & 0,917 & 0,910 & 0,957 & - \\
Eficiência Interorganizacional & 0,951 & 0,983 & 0,987 & 0,823 \\
Fornecedores & 0,659 & 0,831 & 0,885 & - \\
Mercado & 0,671 & 0,901 & 0,924 & - \\
Rivalidade Competitiva & 0,784 & 0,867 & 0,916 & - \\
\hline
\end{tabular}

Fonte: Dados da pesquisa (2012)

Obs.: Variáveis independentes não possuem $\mathrm{R}^{2}$.

Após a análise das variâncias médias extraídas e dos índices de confiabilidade, seguiu-se com a validação discriminante das VLs segundo o critério de Fornell e Lacker (1981) $\left({ }^{\sqrt{A V E}>}\right.$ correlações de Pearson com as outras VLs). 
Observa-se, na Tabela 7, que as ${ }^{\sqrt{A V E}}$ de todas as VLs são maiores que as respectivas correlações de Pearson com as outras VLs, mostrando boa discriminação para cada constructo.

Tabela 7 - Valores de qualificação discriminante do modelo estrutural

\begin{tabular}{llllll}
\hline Dimensões & 1 & 2 & 3 & 4 & 5 \\
\hline 1. Clientes e Consumidores & 0,958 & & & & \\
2. Eficiência Interorganizacional & 0,707 & 0,975 & & & \\
3. Fornecedores & 0,496 & 0,708 & 0,812 & & \\
4. Mercado & 0,667 & 0,883 & 0,724 & 0,819 & \\
5. Rivalidade Competitiva & 0,820 & 0,709 & 0,610 & 0,630 & 0,885 \\
\hline
\end{tabular}

Fonte: Dados da pesquisa (2012)

Obs.: os valores em negrito representam a $\sqrt{A V E}$ da VL. Os outros valores são as correlações de Pearson entre cada variável.

Continuando com a validação do modelo, avaliaram-se médias entre valores originais e valores de reamostragem por meio da consecução de testes t de Student para cada VL e VO, devendo estes serem maiores que 1,96 (ou ${ }^{p \leq 0,05}$ ). Conforme Tabela 8, apesar da VL Clientes e Consumidores estar próxima ao valor de referência $(0,04)$, ainda está no intervalo de $95 \%$ de confiança, assim como todas as outras VLs e VOs, o que demonstra que as médias dos modelos de mensuração (VOs) e estrutural se encaixam nas médias das reamostragens, evidenciando adequação do modelo.

Tabela 8 - Valores t de Student e p-valores

\begin{tabular}{|c|c|c|c|c|c|}
\hline Variáveis latentes & Teste t & p-valor & Variáveis observadas & Teste $\mathrm{t}$ & p-valor \\
\hline \multirow{3}{*}{ Clientes e Consumidores } & \multirow{3}{*}{2,034} & \multirow{3}{*}{0,04} & $\mathrm{C} 1$ & 97,556 & 0,000 \\
\hline & & & $\mathrm{C} 2$ & 111,804 & 0,000 \\
\hline & & & EIN1 & 231,108 & 0,000 \\
\hline Eficiência & \multirow{4}{*}{ - } & \multirow{4}{*}{-} & EIN2 & 206,518 & 0,000 \\
\hline \multirow[t]{3}{*}{ Interorganizacional } & & & EIN3 & 61,977 & 0,000 \\
\hline & & & EIN4 & 506,055 & 0,000 \\
\hline & & & F3 & 9,903 & 0,000 \\
\hline \multirow{5}{*}{ Fornecedores } & \multirow{5}{*}{2,117} & \multirow{5}{*}{0,03} & F4 & 33,474 & 0,000 \\
\hline & & & F5 & 23,624 & 0,000 \\
\hline & & & F6 & 8,108 & 0,000 \\
\hline & & & M1 & 23,915 & 0,000 \\
\hline & & & M2 & 74,214 & 0,000 \\
\hline \multirow{5}{*}{ Mercado } & \multirow{5}{*}{4,824} & \multirow{5}{*}{0,000} & M3 & 10,350 & 0,000 \\
\hline & & & M4 & 29,556 & 0,000 \\
\hline & & & M5 & 8,499 & 0,000 \\
\hline & & & M6 & 17,815 & 0,000 \\
\hline & & & $\mathrm{RC} 1$ & 44,617 & 0,000 \\
\hline \multirow[t]{2}{*}{ Rivalidade Competitiva } & \multirow[t]{2}{*}{3,057} & \multirow[t]{2}{*}{0,01} & RC2 & 28,663 & 0,000 \\
\hline & & & RC3 & 29,257 & 0,000 \\
\hline
\end{tabular}

Fonte: Dados da pesquisa (2012) 
Empreendeu-se, em seguida, a avaliação da validade preditiva $\left(Q^{2}\right)$ e tamanho de efeito $\left(f^{2}\right)$, considerando-se valores de referência de $Q^{2}>0$ e $f^{2}>0,35$ (HAIR JR. et al., 2014). Em todos os casos, os valores (Tabela 9) atenderam satisfatoriamente aos padrões, demonstrando que o modelo possui boa capacidade preditiva e que as VLs são importantes para ajuste geral do modelo.

Tabela 9 - Valores de validade preditiva $\left(Q^{2}\right)$ e de tamanho do efeito $\left(f^{2}\right)$

\begin{tabular}{lll}
\hline Dimensões & $\mathrm{Q}^{2}$ & $\mathrm{f}^{2}$ \\
\hline Clientes e Consumidores & - & 0,631 \\
Eficiência Interorganizacional & 0,637 & 0,903 \\
Fornecedores & - & 0,428 \\
Mercado & - & 0,534 \\
Rivalidade Competitiva & - & 0,544
\end{tabular}

Fonte: dados da pesquisa (2012)

Finalizando as análises de validação, verificou-se o índice de adequação do modelo estrutural (GoF), tomando-se como valor de referência um GoF > 0,36 conforme proposto por Wetzels et al. (2009 apud BIDO; SILVA; RINGLE, 2014). O GoF encontrado (Tabela 10) foi de 0,796657, evidenciando excelente adequação do modelo estrutural.

Tabela 10 - Índice de adequação do modelo estrutural (GoF)

\begin{tabular}{|c|c|c|c|c|}
\hline Dimensões & Variáveis & AVE & $\begin{array}{l}\text { AVE X } \\
\text { Variáveis }\end{array}$ & $\mathrm{R}^{2}$ \\
\hline Clientes e Consumidores & 2 & 0,917 & 1,834 & \multirow{5}{*}{0,823} \\
\hline Eficiência Interorganizacional & 4 & 0,951 & 3,804 & \\
\hline Fornecedores & 4 & 0,659 & 2,636 & \\
\hline Mercado & 6 & 0,671 & 4,026 & \\
\hline Rivalidade Competitiva & 3 & 0,784 & 2,352 & \\
\hline & & & AVE Médio & $\mathrm{R}^{2}$ Médio $\left({ }^{\star *}\right)$ \\
\hline & & & $\frac{0,771158}{15}$ & 0,823 \\
\hline
\end{tabular}

Fonte: dados da pesquisa (2012)

Obs.: $\quad\left({ }^{*}\right)$ Média ponderada pelo número de variáveis. $\left(^{* *}\right)$ Média aritmética

Assim, chega-se ao modelo final para Eficiência Interorganizacional, apresentado na Figura 3 , com suas respectivas cargas fatoriais $(\lambda)$, betas das regressões lineares $(\beta)$ e coeficientes de determinação $\left(R^{2}\right)$. 
Figura 3 - Modelo estrutural ajustado sobre Eficiência Interorganizacional

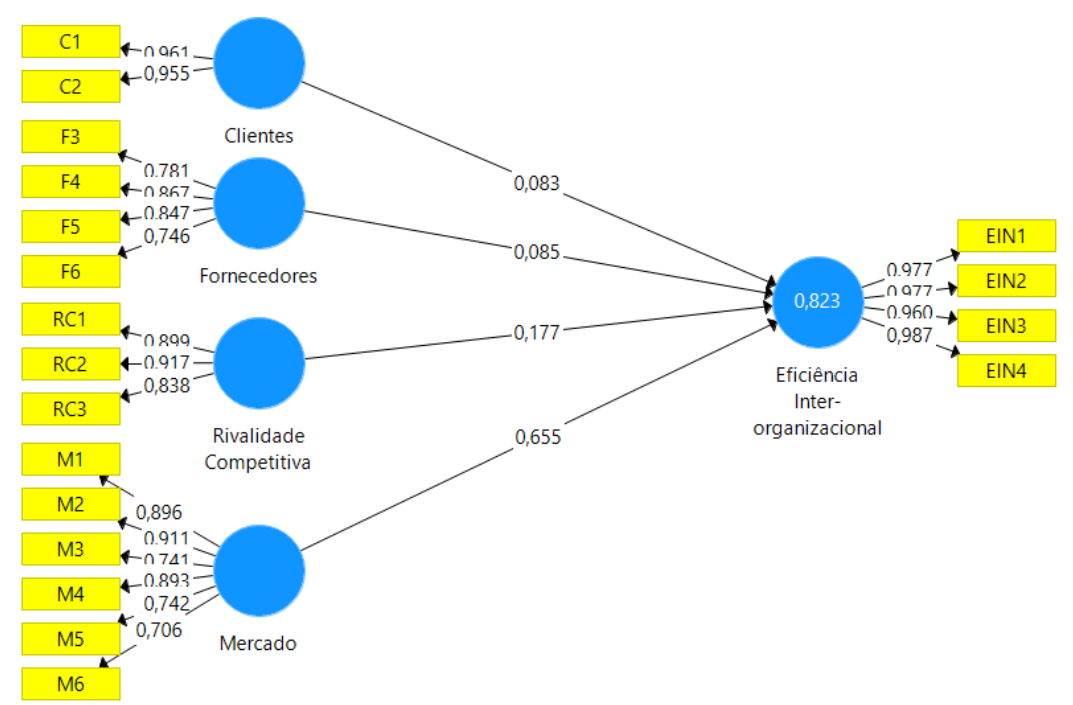

Fonte: Dados da pesquisa (2012)

\section{DISCUSSÃO DOS RESULTADOS}

Retomando o objetivo deste estudo, buscou-se verificar se dimensões de clientes e consumidores, fornecedores, mercado e rivalidade competitiva estão positivamente relacionadas a dimensões de eficiência e eficácia organizacional e eficiência interorganizacional, quando todas são assistidas por sistemas de ERP.

Os quadros 2 e 3 resumem e apresentam estas relações e testam as hipóteses do estudo.

Quadro 2 - Análise das hipóteses de relações estruturais com Eficiência e Eficácia Organizacional

\begin{tabular}{|l|l|l|l|l|l|l|}
\hline Hipóteses & Dimensões & $\begin{array}{l}\text { Efeito } \\
\text { hipotético }\end{array}$ & $\begin{array}{l}\text { Efeito } \\
\text { observado }\end{array}$ & $\begin{array}{l}\beta \text { do } \\
\text { caminho }\end{array}$ & $\begin{array}{l}p- \\
\text { valor }\left({ }^{*}\right)\end{array}$ & Resultado \\
\hline H1 & $\begin{array}{l}\text { Clientes e } \\
\text { Consumidores }\end{array}$ & Positivo & $\begin{array}{l}\text { Positivo } \\
\text { médio }\end{array}$ & 0,522 & 0,000 & $\begin{array}{l}\text { Confirma } \\
\text { H1 }\end{array}$ \\
\hline H2 & Fornecedores & Positivo & Positivo fraco & 0,198 & 0,028 & $\begin{array}{l}\text { Confirma } \\
\text { H2 }\end{array}$ \\
\hline H3 & Mercado & Positivo & Positivo fraco & 0,393 & 0,000 & $\begin{array}{l}\text { Confirma } \\
\text { H3 }\end{array}$ \\
\hline H4 & Rivalidade Competitiva & Positivo & $\begin{array}{l}\text { Negativo } \\
\text { fraco }\end{array}$ & $-0,104$ & 0,024 & Rejeita H4 \\
\hline
\end{tabular}

Fonte: dados da pesquisa (2012)

Obs.: $\left.{ }^{*}\right)$ valores significativos com $p \leq 0,05$.

De acordo com os resultados apresentados no Quadro 2, verificou-se que as dimensões "clientes e consumidores", "fornecedores" e "mercado" estão positivamente relacionadas com eficiência e eficácia organizacional, confirmando as hipóteses $\mathrm{H} 1, \mathrm{H} 2$ e H3, apesar das relações serem consideradas baixas. Entretanto, 
a dimensão rivalidade competitiva apresentou associação negativa, sendo a hipótese $\mathrm{H} 4$ rejeitada.

Quadro 3 - Análise das hipóteses de relações estruturais com Eficiência Interorganizacional

\begin{tabular}{|l|l|l|l|l|l|l|}
\hline Hipóteses & Dimensões & $\begin{array}{l}\text { Efeito } \\
\text { hipotético }\end{array}$ & $\begin{array}{l}\text { Efeito } \\
\text { observado }\end{array}$ & $\begin{array}{l}\beta \text { do } \\
\text { caminho }\end{array}$ & $\begin{array}{l}p- \\
\text { valor( }\end{array}$ & Resultado \\
\hline H1 & $\begin{array}{l}\text { Clientes e } \\
\text { Consumidores }\end{array}$ & Positivo & Positivo fraco & 0,083 & 0,041 & $\begin{array}{l}\text { Confirma } \\
\text { H5 }\end{array}$ \\
\hline H2 & Fornecedores & Positivo & Positivo fraco & 0,085 & 0,032 & $\begin{array}{l}\text { Confirma } \\
\text { H6 }\end{array}$ \\
\hline H3 & Mercado & Positivo & Positivo fraco & 0,177 & 0,000 & $\begin{array}{l}\text { Confirma } \\
\text { H7 }\end{array}$ \\
\hline H4 & Rivalidade Competitiva & Positivo & $\begin{array}{l}\text { Positivo } \\
\text { médio }\end{array}$ & 0,655 & 0,011 & $\begin{array}{l}\text { Confirma } \\
\text { H8 }\end{array}$ \\
\hline
\end{tabular}

Fonte: dados da pesquisa (2012)

Obs.: $\left.{ }^{*}\right)$ valores significativos com $p \leq 0,05$.

Com $\beta$ um pouco maiores, mas ainda considerados baixos, as dimensões de "clientes e consumidores", "fornecedores", "mercado" e "rivalidade competitiva" estão positivamente relacionadas à dimensão estratégica de "eficiência interorganizacional", comprovando as hipóteses H5, H6, H7 e H8.

De forma geral, estes resultados conseguem demonstrar que há relações positivas entre sistemas de ERP e dimensões estratégicas de resultado, conforme levantado por Hedman e Borell (2002) e Sedera, Gable e Chan (2003). Em especial, verifica-se maior relação entre a dimensão "clientes e consumidores" e "eficiência e eficácia organizacional" $(0,522)$ e entre "rivalidade competitiva" e "eficiência interorganizacional" $(0,655)$, sendo que a primeira pode estar ligada às funções de vendas e suporte. Contudo, apesar de os resultados gerais contribuírem para reforçar a tese de maturidade organizacional (CHAN, SABHERWAL; TATCHER, 2006; LÖBLER; BOBSIN; VISENTINI, 2008) para a obtenção de benefícios de mais longo prazo como os estratégicos indicados pelos baixos $\beta$ de caminho, a relação mais forte entre rivalidade competitiva e eficiência interorganizacional vai na contramão desta tese. Este resultado merece melhor investigação em futuros estudos.

\section{CONCLUSÃO}

É relevante afirmar que, neste estudo, as dimensões estratégicas, auxiliadas por sistemas de ERP, de clientes e consumidores, fornecedores, mercado e 
rivalidade competitiva foram definidas como preditoras das dimensões estratégicas de resultados de eficiência e eficácia organizacional e eficiência interorganizacional.

Desta forma, quanto maior a contribuição dos sistemas de ERP nas dimensões preditoras, maior a contribuição destes mesmos sistemas para as dimensões de eficiência e eficácia organizacional e de eficiência interorganizacional. Essa relação, apesar de ser comprovada de forma tímida, soma esforços com os resultados apontados por Mahmood e Soon (1991) e Saccol et al. (2004), contribui, em alguma intensidade e de forma empírica, para a demonstração da relação entre sistemas de ERP e efetividade organizacional apontada por Hedman e Borell (2002) e Sedera, Gable e Chan (2003) e preenche, em parte, lacunas desta relação para pequenas e médias empresas (MENDES; ESCRIVÃO FILHO, 2000; 2002).

Como as relações se demonstram positivas, mas fracas e como a dimensão de rivalidade competitiva obteve relação ligeiramente negativa com a dimensão de eficiência e eficácia organizacional, reforça-se a tese da necessidade de maturidade da empresa para extrair mais benefícios de sistemas de informação e, semelhantemente, de sistemas de ERP (CHAN, SABHERWAL; TATCHER, 2006; LÖBLER; BOBSIN; VISENTINI, 2008).

Os principais limitantes do estudo estão, efetivamente, no tamanho e localização das amostras e no tempo de implantação (média de 2,32 anos). Estes limitantes não permitem a identificação de alterações no uso dos sistemas de ERP que, potencialmente, podem ocorrer no longo prazo. Desta forma, não é possível fazer generalizações para pequenas e médias empresas, devendo ser considerados os resultados como característicos da região de Patos de Minas.

Recomendam-se outros estudos com amostras mais representativas, além de um levantamento mais profundo e/ou maior segmentação de outras dimensões preditivas da eficiência e eficácia organizacional e interorganizacional.

\section{REFERÊNCIAS}

BAKOS, J. Y.; TREACY, M. Information technology and corporate strategy: a research perspective. MIS Quarterly, v. 10, n. 2, pp. 107-119, Jun. 1986.

http://dx.doi.org/10.2307/249029

BARROS, F. Reestruturação no mercado de software de gestão, 06 fev. 2003. Disponível em: 
<http://computerworld.uol.com.br/AdPortalv5/adCmsDocumentShow.aspx?GUID=6D926CC6 -9137-458F-A687-6ECC77A6FBCC\&ChannelID=21 >. Acesso em: 02 out. 2014.

BECKER, J. L.; LUNARDI, G. L.; MAÇADA, A. C. G. Análise de eficiência dos bancos brasileiros: um enfoque nos investimentos realizados em tecnologia da informação. In: ENCONTRO NACIONAL DE ENGENHARIA DE PRODUÇÃO, 22., 2002, Curitiba. Anais... Rio de Janeiro: ABEPRO, 2002. http://dx.doi.org/10.1590/S0103-65132003000200007

BERNROIDER, E; KOCH, S. Decision making for ERP: investments from the perspective of organizational impact: preliminary results from an empirical study. In: AMERICAS' CONFERENCE ON INFORMATION SYSTEMS, 5., 2010, Milwaukee. Anais... Illinois: Association for Information Systems, 1999.

BORT, S.; KIESER, A. Fashion in organization theory: an empirical analysis of the diffusion of theoretical concepts. Organization Studies, v. 32, n. 5, pp. 655-681, 2011. http://dx.doi.org/10.1177/0170840611405427

BRYNJOLFSSON, R. The productivity paradox of information technology. Communications of the ACM, v. 36, n. 12, pp. 67-77, 1993. http://dx.doi.org/10.1145/163298.163309

CARR, N. G. IT doesn't matter. Harvard Business Review, v. 81, n. 5, pp. 41-48, May 2003.

CHAN, Y. E.; SABHERWAL, R.; THATCHER, J. B. Antecedents and outcomes of strategic IS alignment: an empirical investigation. IEEE Transactions on Engineering Management, v. 51, n. 3, pp. 27-47, 2006. http://dx.doi.org/10.1109/TEM.2005.861804

CLEMONS, E. Information systems for sustainable competitive advantage. Information \& Management, v. 11, n. 3, pp. 131-136, Nov. 1986. http://dx.doi.org/10.1016/03787206(86)90010-8

DAVENPORT, T. H. Putting the enterprise into the enterprise system. Harvard Business Review, pp. 1221-1231, Jul./Aug. 1998.

FELICIANO, S.; MAÇADA, A. C. G. Impactos da consumerização de TI no desempenho e na governança de TI. In: INTERNATIONAL CONFERENCE ON INFORMATION RESOURCES MANAGEMENT, 6., 2013, Natal. Anais... Illinois: Association for Information Systems, 2013.

FERREIRA, L. B.; RAMOS, A. S. M. Tecnologia da informação: commodity ou ferramenta estratégica? Revista de Gestão da Tecnologia e Sistemas de Informação, v. 2, n. 1, p. 69-79, 2005. http://dx.doi.org/10.1590/S1807-17752005000100006

FICHMAN, R. G. Going beyond the dominant paradigm for information technology innovation research: emerging concepts and methods. Journal of the Association for Information Systems, v. 5, n. 8, pp. 314-355, 2004.

FORNELL, C.; LARCKER, D. F. Evaluating structural equation models with unobservable variables and measurement error. Journal of Marketing Research, v. 18, n. 1, pp. 39-50, 1981. http://dx.doi.org/10.2307/3151312

GRAEML, A. R. As ideias com as quais se pensa na avaliação de projetos de tecnologia da informação. In: ENCONTRO NACIONAL DE ENGENHARIA DE PRODUÇÃO, 23., 1998, Niterói. Anais... Rio de Janeiro: ABEPRO, 1998. 
HAIR JR., J. F.; HULT, T. M.; RINGLE, C. M.; SARSTEDT, M. A primer on partial least squares structural equation modeling (PLS-SEM). Los Angeles: SAGE, 2014.

HEDMAN, J.; BORELL, A. The impact of enterprise resource planning systems on organizational effectiveness: an artifact evaluation. In: NAH, F. F. Enterprise resource planning solutions \& management. Hershey: IRM Press, 2002.

HENSELER, J.; RINGLEAND, C. M.; SINKOVICS, R. R. The use of partial least squares path modeling in international marketing. In: SINKOVICS, R. R.; GHAURI, P. N. (Eds.). New challenges to international marketing. [S.I.]: Emerald Group Publishing, 2009. (Advances in international marketing, 20). Chapter 12 (Part IV). pp. 277-319.

KUMAR K.; VAN HILLEGERSBERG J. Enterprise resource planning: introduction.

Communications of the ACM, New York, v. 43, n. 4, pp. 22-26, Apr. 2000.

http://dx.doi.org/10.1145/332051.332063

LAUDON, K. C.; LAUDON, J. P. Sistemas de informação gerenciais: administrando a empresa digital. 5. ed. São Paulo: Pearson Prentice-Hall, 2004.

LAURINDO, F. J. B. Tecnologia da informação: eficácia nas organizações. São Paulo: Futura, 2002.

LEE, J.; COLLAR, E. Information technology fashions: lifecycle phase analysis. In: ANNUAL HAWAII INTERNATIONAL CONFERENCE ON SYSTEM SCIENCES, 36., 2003, Hawaii.

Anais... New York: IEEE, 2003. http://dx.doi.org/10.1109/HICSS.2003.1174782

LÖBLER, M. L.; BOBSIN, D.; VISENTINI, M. S.; Alinhamento entre o plano de negócio e o plano de tecnologia de informação das empresas: análise comparativa através dos níveis de maturidade e fatores críticos de sucesso. Revista de Gestão da Tecnologia e Sistemas de Informação, v. 5, n. 1, p. 37-60, 2008. http://dx.doi.org/10.4301/S1807-17752008000100003

LUFTMAN, J. N.; LEWIS, P. R.; OLDACH, S. H. Transforming the enterprise: the alignment of business and information technology strategies. IBM Systems Journal, v. 32, n. 1, pp. 198-221, 1983. http://dx.doi.org/10.1147/sj.321.0198

MACÊDO, D. G.; GAETE, L.; JOIA, L. A. Análise dos antecedentes à resistência a sistemas empresariais sob a ótica dos gestores de TI. Revista de Administração Contemporânea RAC [Online], Curitiba, v. 18, n. 2, p. 139-160, mar./abr. 2014. http://dx.doi.org/10.1590/S1415-65552014000200003

MAHMOOD, M. A. How information technology resources affect organizational performance and productivity. Information Resources Management Journal, v. 10, Preface, pp. 4-5, 1997.

MAHMOOD, M. A.; SOON, S. K. A comprehensive model for measuring the potential impact of information technology on organizational strategic variables. Decision Sciences, v. 22, n. 4, p. 869-897, Sep. 1991. http://dx.doi.org/10.1111/j.1540-5915.1991.tb00368.x

MENDES, J. V.; ESCRIVÃO FILHO, E. O sistema integrado de gestão é fator de competitividade para as pequenas e médias empresas? Revista Uniara, n. 8, p. 13-21, 2000. 
Sistemas integrados de gestão ERP em pequenas empresas: um confronto entre o referencial teórico e a prática empresarial. Gestão \& Produção, v. 9, n. 3, p. 277-296, dez. 2002. http://dx.doi.org/10.1590/S0104-530X2002000300006

MESQUITA, R. V. Yankee analisa a nova PeopleSoft. ITWeb, 2003. Disponível em: $<$ http://www.itweb.com.br>. Acesso em: 27 set. 2014.

O’BRIEN, J. A. Sistemas de informação: e as decisões gerenciais na era da Internet. 2. ed. São Paulo: Saraiva, 2002.

PARSONS, G. L. Information technology: a new competitive weapon. Sloan Management Review, v. 25, n. 1, pp. 107-119, 1983.

PORTER, M. E. How competitive forces shape strategy. Harvard Business Review, v. 57, n. 2, pp. 137-145, Mar./Apr. 1979.

SACCOL, A. Z.; PEDRON, C. D.; LIBERALI NETO, G.; MACADAR, M. A.; CAZELLA, S. C. Avaliação do impacto dos sistemas ERP sobre variáveis estratégicas de grandes empresas no Brasil. Revista de Administração Contemporânea, v. 8, n. 1, p. 09-34, jan./mar. 2004. http://dx.doi.org/10.1590/S1415-65552004000100002

SANTOS, A. A. Informática na empresa. 5. ed. São Paulo: Atlas, 2010. Cap. 6. p. 147-160.

Informática na empresa. 5. ed. São Paulo: Atlas, 2010. Cap. 7. p. 162-174.

SANTOS, A. M. dos; MAÇADA, A. C. G. Comportamento mimético no abandono de sistemas ERP: o caso de uma organização brasileira. In: AMERICAS' CONFERENCE ON INFORMATION SYSTEMS, 16., 2010, Lima, Peru. Anais... Illinois: Association for Information Systems, 2010.

SCHEER, A.-W.; HABERMANN F. Enterprise resource planning: making ERP a success. Communications of the ACM, New York, v. 43, n. 4, pp. 57-61, Apr. 2000. http://dx.doi.org/10.1145/332051.332073

SEDERA, D.; GABLE, G.; CHAN, T. Measuring enterprise systems success: a preliminary model. In: AMERICAS' CONFERENCE ON INFORMATION SYSTEMS, 9., 2003, Tampa. Anais... Illinois: Association for Information Systems, 2003.

SHERMAN, E. ERP attitude adjustments. ComputerWorlds, Framingham, pp. 52-53, Feb. 2000.

SOUZA, C. A.; ZWICKER, T. Ciclo de vida de sistemas ERP. Caderno de Pesquisas em Administração, São Paulo, v. 1, n. 11, 2000.

SOUZA, P. M.; VASCONCELOS, M. C. R. L.; TAVARES, M. C.; CARVALHO, R. B.; GUIMARÃES, E. R. Contribuições dos sistemas Enterprise Resource Planning para a gestão da informação e do conhecimento: um estudo em uma empresa de pequeno porte na área gráfica. Perspectivas em Gestão \& Conhecimento, João Pessoa, v. 3, n. esp., p. 109-127, out. 2013.

STAMFORD, P.P. ERP: prepare-se para esta mudança. Knowledge Management Press \& Consulting, 15 jun. 2003. Seção Artigos e Clippings. Disponível em:

<http://www.kmpress.com.br/portal/artigos/preview.asp?id=134>. Acesso em: 30 jun. 2014. 
SWIFT, R. CRM, Customer relationship management: o revolucionário marketing de relacionamento com os clientes. 9. ed. Rio de Janeiro: Elsevier, 2001.

TENENHAUS, M.; VINZI, V. E.; CHATELIN, Y.; LAURO, C. PLS path modeling. Computational Statistics \& Data Analysis, v. 48, n. 1, pp. 159-205, 2005. http://dx.doi.org/10.1016/i.csda.2004.03.005

THOMPSON; A.; STRICKLAND, A. J. Planejamento estratégico: elaboração, implementação e execução. São Paulo: Pioneira, 2002.

WANG, P. Chasing the hottest IT: effects of information technology fashion on organizations. MIS Quarterly, v. 34, n. 1, pp. 63-85, 2010.

What drives waves in information technology? IT discourse from the organizing vision perspective. In: INTERNATIONAL CONFERENCE ON INFORMATION SYSTEMS, 22., 2001, New Orleans. Anais... Los Angeles: University of California, 2001.

WEITZEN, H. S. O poder da informação: como transformar a informação que você domina em um negócio lucrativo. São Paulo: McGraw-Hill, 1991.

WOOD JR., T. Modas e modismos gerenciais: o caso dos sistemas integrados de gestão. Série Relatórios de Pesquisa, NPP, Núcleo de Pesquisas e Publicações. Escola de Administração de Empresas de São Paulo, FGV. Relatório №. 16, 1999.

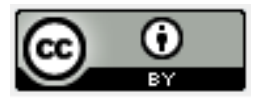

Artigo recebido em 15/12/2014 e aceito para publicação em 20/11/2015 DOI: http://dx.doi.org/ 10.14488/1676-1901.v15i4.1954 